\title{
Design of polarimeters based on liquid crystals and biaxial crystals for polarization metrology
}

\section{Diseño de polarímetros basados en cristales líquidos y cristales biáxicos para la metrología de polarización}

\author{
A. Peinado ${ }^{1, \mathrm{~S}^{*}}$, A. Lizana ${ }^{1, \mathrm{~S}}$, J. Campos ${ }^{1, \mathrm{~S}}$ \\ 1. Departament de Física, Universitat Autònoma de Barcelona, 08193, Bellaterra, Spain \\ ${ }^{(*)}$ E-mail: alba.peinado@uab.es \\ S: SEDOPTICA member
}

DOI: $10.7149 /$ OPA.49.4.49501

\begin{abstract}
:
This paper reviews three polarimeters based on different types of liquid crystal cells (parallel and twisted nematic, and ferroelectric), as well as a polarimeter based on the conical refraction phenomenon (occurring in biaxial crystals). A comparative analysis between them is included in order to summarize their main features and to point out their strengths and limitations. Then, the best candidate for imaging polarimetry is selected and implemented. Several polarization images are analyzed in order to show the potential of the instrument. Moreover, a new experimental configuration is presented, which combines an imaging polarimeter and a module used to achieve sub-pixelresolution in a system where the resolution is limited by detector pixel size. Experimental results show an improvement in the spatial resolution of polarization images by a factor of 1.4.
\end{abstract}

Key words: polarimetry, liquid crystals, conical refraction, image polarimeter, super-resolution.

\section{RESUMEN:}

Este artículo revisa tres polarímetros basados en diferentes tipos de láminas de cristales líquidos (nemático con estructura paralela y helicoidal, y ferroeléctrico), así como un polarímetro basado en el fenómeno de la refracción cónica (que ocurre en cristales biaxiales). Se incluye un análisis comparativo entre ellos resumiendo sus principales características y señalando sus puntos fuertes y limitaciones. A continuación, se selecciona el mejor candidato para la polarimetría de imagen y es implementado. Se analizan varias imágenes de polarización con el fin de mostrar el potencial del instrumento. Además, se presenta una configuración experimental nueva, que combina un polarímetro de imagen y un módulo utilizado para lograr resolución sub-píxel en un sistema en el que la resolución está limitada por el tamaño del píxel del detector. Los resultados experimentales muestran una mejora en la resolución espacial de las imágenes de polarización en un factor de 1.4.

Palabras clave: polarimetría, cristales líquidos, refracción cónica, polarímetro de imagen, superresolución.

\section{REFERENCES AND LINKS}

[1] N. Ghosh, and I. A. Vitkin, "Tissue polarimetry: concepts, challenges, applications, and outlook," J. Biomed. Opt. 16, 10801-110801-29 (2011).

[2] A. Pierangelo, A. Benali, M.-R. Antonelli, T. Novikova, P. Validire, B. Gayet, and A. De Martino, "Ex-vivo characterization of human colon cancer by Mueller polarimetric imaging," Opt. Express 19, 1582-1593 (2011).

https://doi.org/10.1364/OE.19.001582

[3] D. Gisler, H. M. Schmid, C. Thalmann, H. P. Povel, J. O. Stenflo, F. Joos, et al., "CHEOPS/ZIMPOL: a VLT instrument study for the polarimetric search of scattered light from extrasolar planets," Proc. SPIE 5492, 463-474 (2004) https://doi.org/10.1117/12.550366 
[4] S. V. Berdyugina, A. V. Berdyugin, D. M. Fluri, and V. Piirola, "First Detection of Polarized Scattered Light from an Exoplanetary Atmosphere," Astrophys. J. 673, L83-L86 (2008). https://doi.org/10.1086/527320

[5] A. Peinado, "Design of polarimeters based on liquid crystals and biaxial crystals for polarization metrology," Doctoral thesis (2014). http://ddd.uab.cat/record/128800

[6] A. Peinado, A. Lizana, J. Vidal, C. Iemmi, and J. Campos, "Optimization and performance criteria of a Stokes polarimeter based on two variable retarders," Opt. Express 18, 9815-9830 (2010). https://doi.org/10.1364/OE.18.009815

[7] A. Peinado, A. Lizana, J. Vidal, C. Iemmi, and J. Campos, "Optimized Stokes polarimeters based on a single twisted nematic liquid-crystal device for the minimization of noise propagation," Appl. Opt. 50, 5437-5445 (2011). https://doi.org/10.1364/A0.50.005437

[8] A. Peinado, A. Lizana, and J. Campos, "Optimization and tolerance analysis of a polarimeter with ferroelectric liquid crystals," Appl. Opt. 52, 5748-5757 (2013). https://doi.org/10.1364/A0.52.005748

[9] A. Peinado, A. Turpin, A. Lizana, E. Fernández, J. Mompart, and J. Campos, "Conical refraction as a tool for polarization metrology," Opt. Lett. 38, 4100-4103 (2013). https://doi.org/10.1364/OL.38.004100

[10] A. Peinado, A. Lizana, A. Turpín, C. Iemmi, T. K. Kalkandjiev, J. Mompart, and J. Campos, "Optimization, tolerance analysis and implementation of a Stokes polarimeter based on the conical refraction phenomenon," Opt. Express 23, 5636-5652 (2015). https://doi.org/10.1364/OE.23.005636

[11] R. A. Chipman, "Polarimetry," in Handbook of Optics, 2nd ed. (McGraw-Hill, 1995).

[12] A. Lizana, I. Estévez, A. Turpin, C. Ramirez, A. Peinado, and J. Campos, "Implementation and performance of an in-line incomplete Stokes polarimeter based on a single biaxial crystal," Appl. Opt. 54, 8758-8765 (2015). https://doi.org/10.1364/A0.54.008758

[13] P. Taylor, Theory and Applications of Numerical Analysis, 2nd ed. (Academic Press, 1996).

[14] J. S. Tyo, "Design of Optimal Polarimeters: Maximization of Signal-to-Noise Ratio and Minimization of Systematic Error," Appl. Opt. 41, 619-630 (2002). https://doi.org/10.1364/A0.41.000619

[15] M. H. Smith, J. B. Woodruff, and J. D. Howe, "Beam wander considerations in imaging polarimetry," Proc. SPIE 3754, 50-54 (1999). https://doi.org/10.1117/12.366359

[16] J. S. Tyo, D. L. Goldstein, D. B. Chenault, and J. A. Shaw, "Review of passive imaging polarimetry for remote sensing applications," Appl. Opt. 45, 5453-5469 (2006). https://doi.org/10.1364/A0.45.005453

[17] S. Aïnouz, J. Zallat, A. de Martino, and C. Collet, "Physical interpretation of polarization-encoded images by color preview," Opt. Express 14, 5916-5927 (2006). https://doi.org/10.1364/0E.14.005916

[18] A. Peinado, A. Lizana, C. Iemmi, and J. Campos, "Polarization imaging with enhanced spatial resolution," Opt. Commun. 338, 95-100 (2015). https://doi.org/10.1016/j.optcom.2014.09.079

[19] A. Márquez, I. Moreno, C. Iemmi, A. Lizana, J. Campos, and M. J. Yzuel, "Mueller-Stokes characterization and optimization of a liquid crystal on silicon display showing depolarization," Opt. Express 16, 16691685 (2008). https://doi.org/10.1364/OE.16.001669

[20] C. Ramirez, B. Karakus, A. Lizana, and J. Campos, "Polarimetric method for liquid crystal displays characterization in presence of phase fluctuations," Opt. Express 21, 3182-3192 (2013). https://doi.org/10.1364/OE.21.003182

[21] J. C. Russ, The Image Processing Handbook (CRC Press, 2011).

[22] R. C. Gonzalez and R. E. Woods, Digital Image Processing, 3rd ed. (Prentice Hall, 2008).

[23] F. Goudail, "Optimization of the contrast in active Stokes images," Opt. Lett. 34, 121-123 (2009). https://doi.org/10.1364/OL.34.000121 


\section{Introduction}

Over the years, polarization metrology has been widespread used in many and diverse research fields ranging from biological tissue assessment $[1,2]$ to the detection of extra-solar planets $[3,4]$. Due to the great interest of polarization data in such applications, research and development of polarimeters (i.e., the basic instruments for polarization metrology) are still very active with the aim of improving their specifications. Recently, liquid crystal (LC)-based technology has been introduced in polarimeters architectures by taking advantage of its appealing features, such as its capability of dynamically changing the effective refractive index anisotropy, this being done at high velocities. Because architectures based on LC cells have no moving parts, experimental errors related to mechanical movements are avoided, and those due to misalignments may be significantly reduced after an initial calibration.

This work summarizes the results of a doctoral thesis [5] which is framed in the field of polarimetry, it focuses on a comparative of polarimeters based on materials presenting refractive index anisotropy. In particular, three different types of LC cells are used for the design of three LC-based polarimeters: parallel aligned nematic, twisted nematic and ferroelectric. Moreover, it presents a novel polarimeter architecture able to statically measure any state of polarization. This last architecture is based on the conical refraction (CR) phenomenon occurring when a focused Gaussian light beam propagates along one of the optical axes of a biaxial crystal. From this comparative, the best candidate for imaging polarimetry is selected and implemented in the laboratory. Next, a detailed analysis of the instrument is given. Finally, it is proposed a new experimental configuration which combines the imaging polarimeter and an extra module used to achieve for the first time, polarimetric sub-pixel-resolution in a system where the resolution is limited by detector pixel size.

(a)

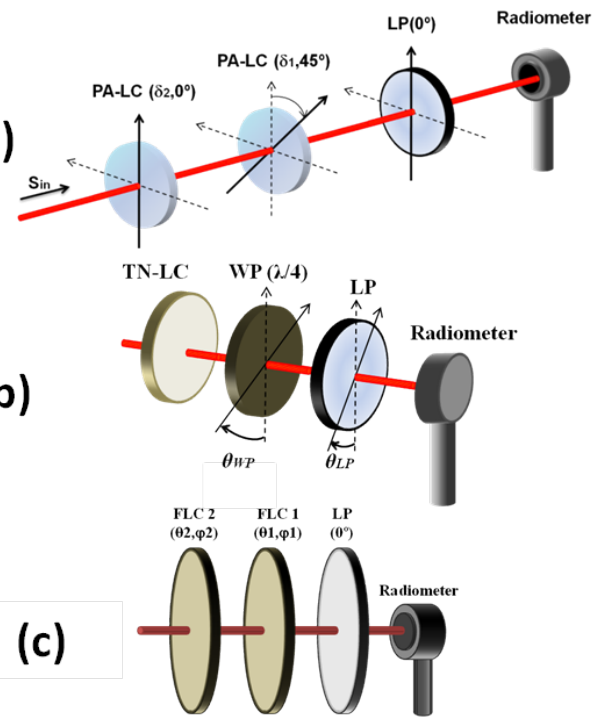

(d)

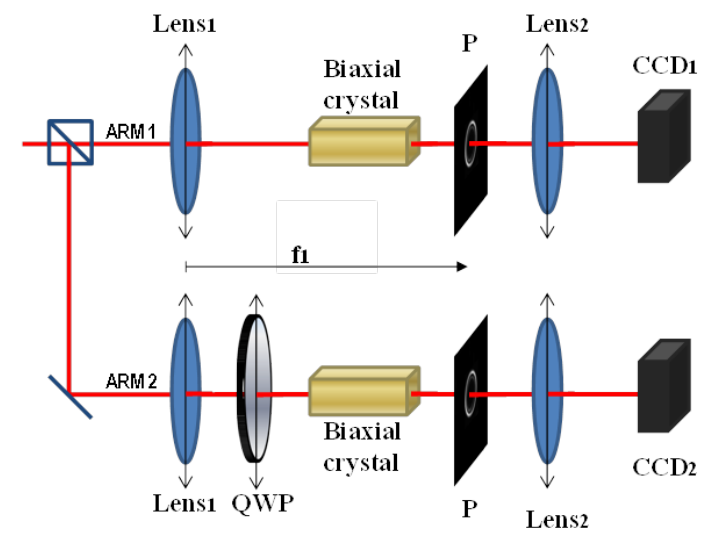

Fig.1. Set-ups of Stokes polarimeters based on: (a) Parallel aligned (PA-LC), (b) twisted nematic (TN-LC) and (c) ferroelectric (FLC) liquid crystal cells, and (d) biaxial crystals

\section{Comparative of polarimeters}

This section aims to review 4 different Stokes polarimeters we have studied in the last few years. First, a polarimeter based on two parallel aligned liquid crystal cells (PA-LC), sketched in Fig.1(a) and studied in detail in [6]. PA-LC cell acts as a linear retarder with variable retardance tunable with the applied voltage. Second, a polarimeter which uses a single twisted nematic LC (TN-LC), sketched in Fig.1(b) and analyzed in detail in [7]. TN-LC cell acts as an elliptical retarder, this enabling both introducing a retardance and rotating the polarization ellipse orientation. The third polarimeter, which configuration is shown in Fig.1(c) and studied in deep in [8], uses two ferroelectric liquid crystal cells of retardance $\lambda / 4$ and $\lambda / 2$. FLC cells are modeled as linear retarders with switchable fast axis orientation by changing the sign of the addressed square electrical signal but with fix retardance. Finally, the polarimeter represented in Fig.1(d) is based on two biaxial crystals where the conical refraction (CR) phenomenon is occurring. When light emerges from the biaxial crystal under CR conditions, a ring of light appears whose intensity distribution depends on the 
incident polarization. The proof of concept of polarization metrology using the conical refraction phenomenon was given in [9], whereas a deep study of the polarimeter performance was provided in [10].

Each polarimeter presents different strengths depending on the architecture itself. Thus, the polarimeter choice depends on the particular application where the instrument has to be used, as some features become more relevant than others. Table.1 summarizes some of the features related to the polarimeters sketched in Fig.1. In addition, as a guide for users interested in the implementation of polarimeters, those features are discussed in the following 7 points:

1. Concerning the type of polarimeter architecture [11], polarimeters based on liquid crystal cells (PA-LC, TN-LC, and FLC) are time-division architectures, i.e., the flux measurements are taken sequentially in time when addressing a set of voltages to the LC cells. By contrast, the CR based polarimeter is a division of amplitude architecture, i.e., it uses a beam splitter to divide the incident beam into two sub-beams and later, both beams are projected simultaneously over multiple analyzers of polarization by means of the conical refraction phenomenon.

2. The equipment needed to implement a punctual and complete Stokes polarimeter is listed in the second row of Table.1. The main difference is that PA-LC and FLC-based polarimeters need 2 monopixel LC cells, whereas TN-LC-based polarimeter only requires a single monopixel LC cell. Finally, to achieve a complete polarimeter, the CR polarimeter architecture needs 2 biaxial crystals, a QWP (quarter waveplate), lenses, and 2 CCD cameras. However, if users are only interested in the linear polarization content, the CR polarimeter architecture can be reduced to a single arm [12] with a single biaxial crystal and CCD.

3. For real time polarimetric applications, where the analyzed light rapidly changes its polarization, a snapshot polarimeter is desired (as the CR polarimeter). Otherwise, if the polarization of the incident beam does not change in time, or such change duration is shorter than the measurement time of the polarimeter, a time-sequential polarimeter results in a suitable alternative. In particular, it is important to wait a transitory time while the LC cell changes of configuration. FLC is the fastest LC cell (transitory time of $0.3 \mathrm{~ms}$ ), whereas PA-LC and TN-LC cells are much slower (transitory time of $300 \mathrm{~ms}$ ).

4. LC-based polarimeters are potential architectures to be used for imaging polarimeters. On the contrary, the CR based polarimeter is limited by its own architecture to be a punctual Stokes polarimeter.

5. All four architectures allow implementing complete Stokes polarimeters, although with different degree of optimized performance. This can be compared by looking at the condition number of the system $[11,13]$. Condition number (CN) [13] is a quality indicator which should be minimized when optimizing the polarimeter design. The best optimized polarimeter is the one using PA-LC (actually, it has the minimum CN possible for a polarimeter design [14]), followed by the CR, the FLC and finally the TN-LC-based polarimeter.

6-7. Finally in the last two rows of Table.1, we point out the main strengths and weaknesses of each polarimeter. All 4 prototypes are optical instruments without any moving optical elements and so, they avoid experimental errors associated to misalignments or to beam wander [15]. Furthermore, CR based polarimeter does not have dynamic elements controllable by an electrical signal, avoiding errors from the phase-to-voltage calibration. First, PA-LC-based polarimeter has the best optimization and also allows implementing any set of polarization analyzers thanks to the versatility of its set-up. This fact may be useful when the measurement of a particular Stokes channel is of interest. However, such polarimeter architecture needs of two LC cells and this type of LC is slow when compared with other LC types. The number of LC cells affects the cost of the polarimeter implementation, as well as it is strongly related to the light losses due to the reflection on the surface of the LC cell. Second, TN-LC-based polarimeter uses a single LC cell, which reduces the final cost. Nevertheless, the measurement duration is long because the TN-LC is slow when changing of configuration. Third, FLC-based polarimeter stands out for its very fast measurements, although it needs two LC cells. Finally, CR based polarimeter takes snapshot measurements, allowing to measure varying polarizations, as well as it is possible to increase data redundancy without increasing the measurement time. However, it requires of 2 CCDs to achieve complete polarimetric measurements, and it is not possible to extend to an imaging polarimeter. 
TABLE 1. Comparative table between the different polarimeters studied. (LP: linear polarizer, QWP: quarter waveplate)

\begin{tabular}{|c|c|c|c|c|}
\hline 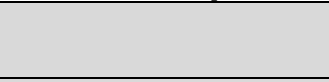 & $\begin{array}{c}\text { PA-LC } \\
\text { polarimeter }\end{array}$ & $\begin{array}{c}\text { TN-LC } \\
\text { polarimeter }\end{array}$ & $\begin{array}{c}\text { FLC } \\
\text { polarimeter }\end{array}$ & $\begin{array}{c}\text { CR } \\
\text { polarimeter }\end{array}$ \\
\hline 1. Type & Division of time & Division of time & Division of time & $\begin{array}{l}\text { Division of } \\
\text { amplitude }\end{array}$ \\
\hline 2. Equipment needed & $\begin{array}{l}\text { 2 PA-LC cells } \\
\text { LP } \\
\text { Radiometer }\end{array}$ & $\begin{array}{l}1 \text { TN-LC cell } \\
\text { (QWP), LP } \\
\text { Radiometer }\end{array}$ & $\begin{array}{c}2 \text { FLC cells } \\
\text { LP } \\
\text { Radiometer }\end{array}$ & $\begin{array}{c}2 \text { biaxial crystals } \\
\text { QWP } \\
2 \text { CCDs, lenses }\end{array}$ \\
\hline 3. Transitory time & $300 \mathrm{~ms}$ & $300 \mathrm{~ms}$ & $0.3 \mathrm{~ms}$ & - \\
\hline 4. Extensible to imaging & Yes & Yes & Yes & No \\
\hline 5. Optimization & $\mathrm{CN}=1.73$ & $\mathrm{CN}=2.69$ & $\mathrm{CN}=2.22$ & $\mathrm{CN}=2.00$ \\
\hline 6. Strengths & $\begin{array}{l}\text { No moving } \\
\text { elements, best } \\
\text { optimization, } \\
\text { versatile }\end{array}$ & $\begin{array}{c}\text { No moving } \\
\text { elements, single } \\
\text { LC cell, } \\
\text { economical }\end{array}$ & $\begin{array}{l}\text { No moving } \\
\text { elements, very } \\
\text { fast }\end{array}$ & $\begin{array}{l}\text { Static set-up, } \\
\text { snapshot } \\
\text { measurements, } \\
\text { high data } \\
\text { redundancy }\end{array}$ \\
\hline 7. Weaknesses & Slow, 2 LC cells & Slow & 2 LC cells & Punctual, 2 CCDs \\
\hline
\end{tabular}

\section{Imaging polarimeter}

After regarding carefully Table.1, we selected the FLC-based polarimeter to be extended to an imaging polarimeter. Not only because of its fast measurements capability and good optimization, but also because of its excellent experimental performance shown in [8] (accuracy in the Stokes elements of 0.05 and repeatability of 0.0002 ).

To adapt the instrument (sketched in Fig.1(c)) to an imaging polarimeter, the radiometer is replaced by a charge-coupled device (CCD) camera. In order to spatially measure the polarization of a plane, a convergent lens (L) images the analyzed scene onto the CCD plane. CCD camera, distributed by Basler, has a resolution of $1000 \times 1000$ pixels $(7.4 \mu \mathrm{m}$ of pixel size).

Two different light sources are tested in the implementation. Firstly, a He-Ne laser (633nm) and secondly, a collimated red light emitting diode (LED, $\lambda_{0}=625 \mathrm{~nm}$ and $\Delta \lambda=17 \mathrm{~nm}$ ). In both cases, a calibration of the system is conducted by taking into account wavelength dependence of polarization properties of the optical elements. We have tested both calibrations by measuring an uniform linear polarized scene. Fig.2 (a) and (b) show the measured Stokes element S0 (corresponding to the total intensity) when illuminating with the laser and the LED, respectively. It is appreciable that when using a very coherent light source (i.e., the laser beam), the total intensity image is degraded by interferential effects, as by the diffraction of dust or small imperfections (as scratches) on the optical elements surfaces. By contrast, when using the LED source, which presents a larger bandwidth, those interferential effects are significantly reduced due to the loss of temporal coherence, and as a consequence, the obtained intensity image is much more uniform. Additionally, we have analyzed the uniformity of the image measurement when regarding to the polarization parameters ellipticity and azimuth. The standard deviation of azimuth and ellipticity are much smaller (one order of magnitude) when using LED compared to the ones obtained with the laser. Therefore, we conclude that the LED is the most suitable light source to illuminate the imaging Stokes polarimeter and it is the illumination source used in the rest of our work.
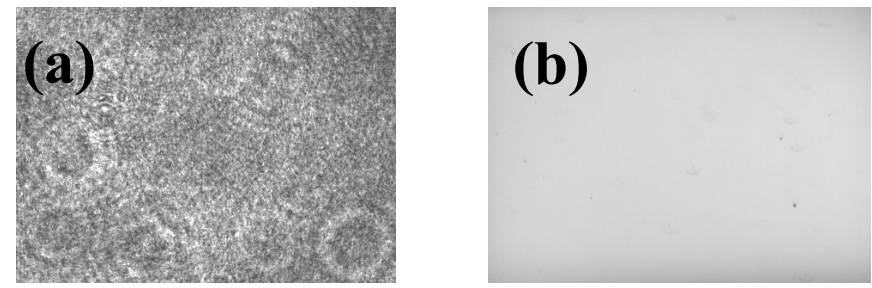

Fig.2. S0 Stokes parameter of a uniform light beam linearly polarized using as light source: (a) He-Ne laser and (b) LED.

Measurements provided by a complete imaging Stokes polarimeter [16] have four channels, these containing the full polarization characterization, typically the four Stokes elements ( $\mathrm{S} 0, \mathrm{~S} 1, \mathrm{~S} 2$ and S3). Thus, the interpretation of this multidimensional content is difficult to grasp at once. We use the method proposed in [17], which represents three polarization parameters in a single synthesized image using the HSV color space. HSV color space represents each color by three attributes: Hue, Saturation and Value. The election of the three polarization parameters (or a combination of them) to be represented in the synthesized image using the HSV model depends on the samples which are being analyzed. The objective of this election is to 
enhance the contrast of the polarization image. In our case, we have used the correspondence between color attribute and polarization parameters shown in Table.2. The main information, azimuth or ellipticity, is encoded with the hue parameter, whereas the total intensity and the DOP modify the pure color by changing the value and saturation, respectively. By using this color correspondence, the interpretation of ellipticity and azimuth images becomes easier because it avoids misinterpretation in particular regions of the image with low intensity values (the color will be darker) or where light contains a significant content of depolarization (the color will be bleached). From now on, we will refer as azimuth HSV image or ellipticity HSV image when we use this HSV model using the color correspondence detailed in Table 2, and the hue encodes the azimuth or the ellipticity, respectively.

TABLE 2. Correspondence between HSV color attributes and polarization parameters used for Stokes measurement images.

\begin{tabular}{|c|c|c|c|}
\hline Color attribute & Color attribute range & Polarization parameter & Polarization parameter range \\
\hline \multirow{2}{*}{ Hue $(\mathrm{H})$} & \multirow{2}{*}{$0^{\circ} \mathbb{C} H \mathbb{C} 360^{\circ}$} & Azimuth $(\chi)$ & $-90^{\circ} \mathbb{C} \chi \mathbb{C} 90^{\circ}$ \\
\hline & & Ellipticity $(\varepsilon)$ & $-45^{\circ} \mathbb{C} \varepsilon \mathbb{C} 45^{\circ}$ \\
\hline Value (V) & $0 \mathbb{C V} \mathbb{C} 1$ & Total intensity (S0) & $0 \mathbb{C}$ S0 $\mathbb{C} 1$ \\
\hline
\end{tabular}

Different polarization scenes have been measured experimentally with the imaging polarimeter. First of all, in order to have a general perspective they are represented with the HSV model and later, specific regions are analyzed in detail by checking a given polarization parameter.

First, we measure the beam transmitted through a polarizer divided into 8 circular sectors, where each sector has a given transmission axis orientation which rotates along $360^{\circ}$. The azimuth and ellipticity HSV images, obtained from the experimental measurements, are represented in Fig.3 (a) and (b), respectively. We observe that the exiting beam has a non-uniform polarization distribution, with 8 distinct circular sectors with different azimuth values distributed along $\left(-90^{\circ}, 90^{\circ}\right)$, labeled with black numbers. In addition, all sectors present a null ellipticity, as we are dealing with linear polarizations. Moreover, the edges between circular sectors are darker because less light is transmitted through these particular regions. In order to see in more detail the uniformity along the circular sectors, we have represented the cross section along the black circle plotted in Fig.3. Azimuth function (Fig.4(a)) is clearly divided into 8 regions with 8 constant values, corresponding to the 8 circular sectors (indicated with the number labels), and there is a link between consecutive regions with intermediate azimuth values. Ellipticity function (Fig.4(b)) keeps a constant value at $0^{\circ}$ (small fluctuations around -1.1 and 1.3), highlighting the linear polarizance of the optical element. Finally, degree of polarization (DOP) function (Fig.4(c)) shows how the light transmitted through the circular sectors is fully polarized ( $D O P=1)$, but light going through the edges is partially polarized (DOP reduced until 0.85 approximately)
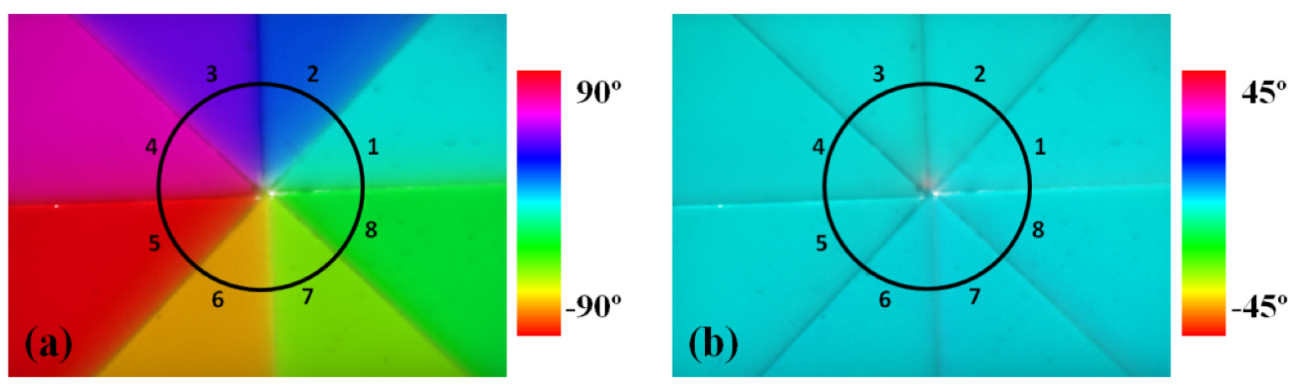

Fig.3. (a) Azimuth HSV image and (b) ellipticity HSV image of a beam transmitted through a polarizer divided into 8 circular sectors with different transmission axis orientation (labeled with a number from 1 to 8). The color legend bar corresponds to the azimuth and ellipticity angle, respectively, encoded by the hue attribute using the HSV model. Cross section of the black circle is in Fig.4.

Second, we analyze the polarization distribution of a linearly polarized beam transmitted through a plastic ruler, being this a polarimetric sample showing spatial variations in the birefringence due to stress. Fig.5(a) and (b) show the azimuth and ellipticity HSV images, respectively. There are four distinct regions labeled with white numbers. Region 1 has a uniform linear polarization (ellipticity is null) corresponding to the light going through a hole of the plastic ruler. Therefore, the light polarization is not altered by the material and so, the measurement in that region corresponds to the polarization of the incident light beam. In region 2 , very little light is transmitted without being deviated through a circular thick flange in the ruler, resulting in a dark area in HSV images. In region 3, there is also a circular flange, although thinner than the one in 
region 2, allowing some light to go through, and several scratches are observed on that surface. Finally, in region 4 few scratches are also observed and this corresponds to the uniform layer of the plastic ruler. Both regions 3 and 4 have a striking color distribution in both polarization HSV images, result of the birefringence stress induced during the manufacturing process of the ruler. In other words, when light is transmitted through this piece of plastic, the exiting beam exhibits a spatial variation in azimuth, as well as in ellipticity according to the non-uniform birefringence stress distribution. In particular, we observe that region 3 presents a faster variation in the spatial distribution of polarization in comparison with region 4 . This is because section 3 presents a larger deformation of the plastic (propinquity of the hole) than section 4 (uniform layer of the ruler), as a stronger stress was applied to generate the flange during the manufacturing process. Furthermore, an analysis of the cross section of the vertical line plotted in Fig.5 is conducted. In particular, we show in Fig. 6 the DOP evolution as a function of the $y$ position along the vertical white line. Region 1 and 4 correspond to fully polarized light (DOP=1), in contrast to regions 2 and 3, where light is partially polarized ( $\mathrm{DOP}<1$ ). The decrease of DOP in these last two regions can be originated by different causes, as for instance, by the diffraction produced by the scratches, by the sharp shape of the plastic in these particular regions, or due to the spatial average of polarizations related to the pixel area.

(a)

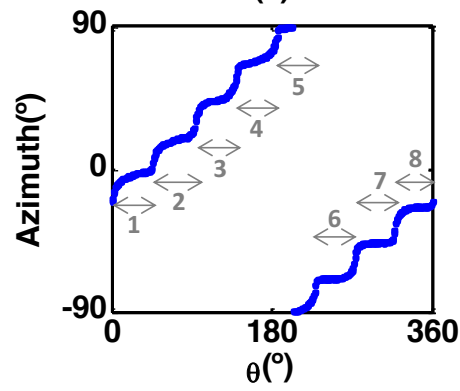

(b)

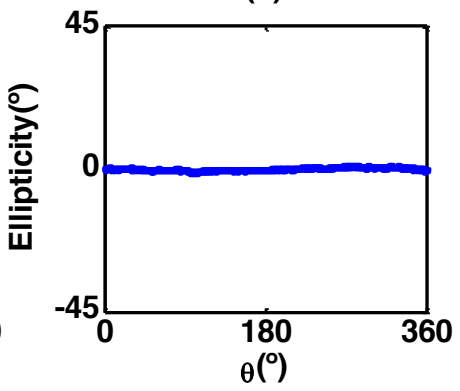

(c)

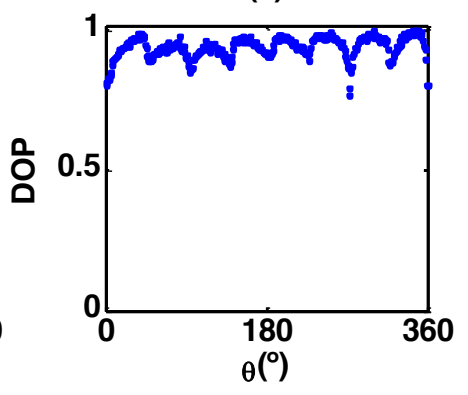

Fig.4. Circular cross section of the Stokes vector measurement shown in Fig.3. (a) Azimuth, (b) ellipticity and (c) DOP as a function of the angle over the circular cross section $(\theta)$. Grey numbers are indicating the 8 distinct regions of the image in Fig.3.
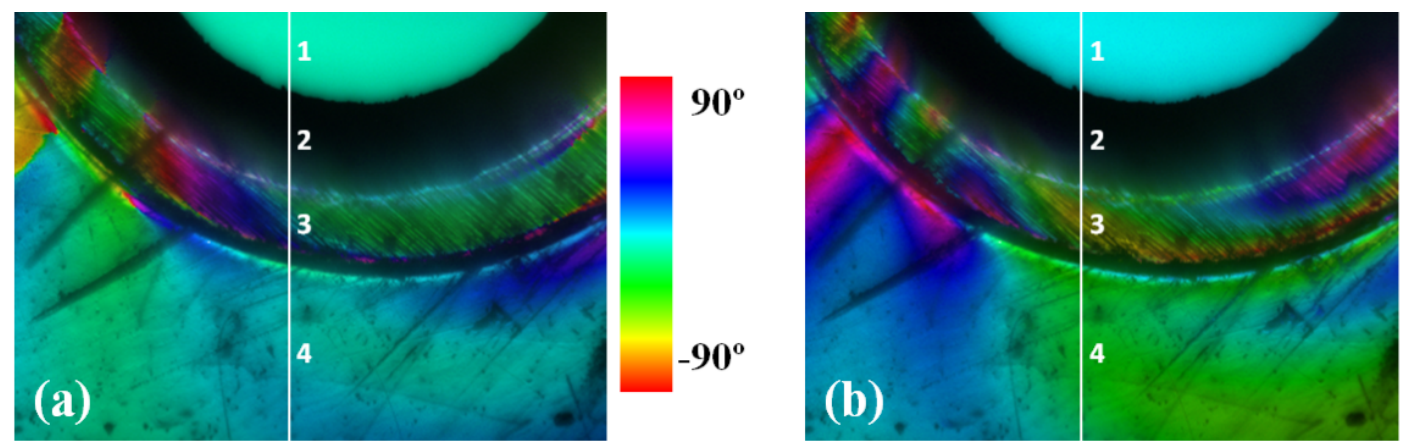

$45^{\circ}$

Fig.5. (a) Azimuth HSV image and (b) ellipticity HSV image of a linearly polarized beam transmitted through a plastic ruler. Numbers are labeling four distinct regions of the image. Cross section of the white line is plotted in Fig,6.

Third, we generate a more pronounced non-uniform polarization distribution by applying an external mechanical strain with a screw on a plastic bar. The beam transmitted through this plastic bar under stress is measured with the imaging Stokes polarimeter. Fig.7 (a) and (b) show the azimuth and ellipticity image, respectively (without using the HSV model to avoid a bleached image related to the low DOP values). We observe a nice banded distribution with rapid changes, more prominent on the left side of both images, due to the birefringence stress. Moreover, it is easy to observe a tendency of the bands wrapping a point on the left side, which actually corresponds to the position where the screw was pressing. Therefore, azimuth and ellipticity images give a fairly accurate picture of the stress distribution in the plastic bar. 


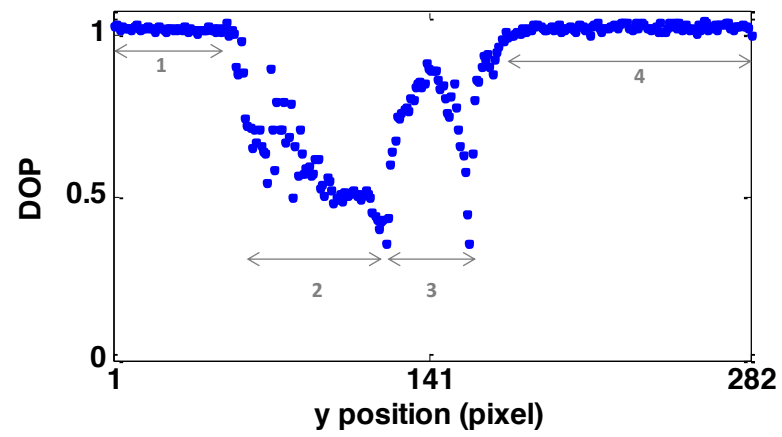

Fig.6. Vertical cross section of the Stokes vector measurement shown in Fig.5. DOP as a function of the position along the $y$ axis. Numbers are indicating the four distinct regions of the image in Fig.5.
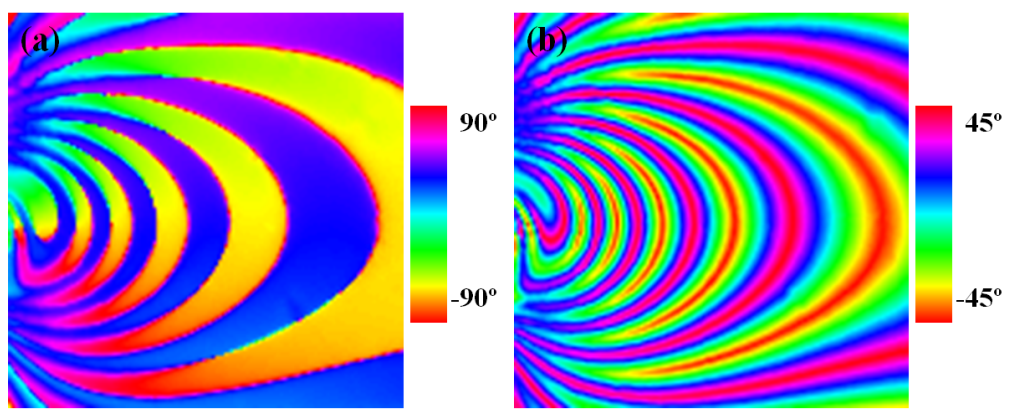

Fig.7. (a) Azimuth and (b) ellipticity image of a linearly polarized beam transmitted through a plastic bar under mechanical stress.

\section{3.a. Super-resolution imaging polarimeter}

In this section, we present an experimental set-up based on LC technology able to provide polarization images with enhanced spatial resolution (SR) [18], when the main limitation in resolution is imposed by the pixel pitch of the CCD camera.

To this aim, the experimental set-up (Fig.8) combines two different modules; a super-resolution module (which reconstructs a single high resolution intensity image of a scene from a set of regular intensity images recorded at lower resolution) with the imaging Stokes polarimeter described in last section (which determines the polarization spatial distribution of a light beam from a set of super-resolved intensity images obtained with the super-resolution module).

The imaging Stokes polarimeter, described in Section 3, obtains polarization images with a spatial resolution (SR) limited by the pixel size of the CCD. Nevertheless, our extended instrument includes an extra module for enhancing the spatial resolution of the intensity images taken by the camera. In this module, light is transmitted by a non-polarizing beam splitter (B-S) towards a parallel aligned liquid crystal on silicon (PA-LCoS) display. The PA-LCoS display used in this work is a PLUTO SLM distributed by HoloEye Systems with a diagonal display of $1.8 \mathrm{~cm}$, a resolution of 1920x1080 pixels, with a pixel pitch of $8 \mu \mathrm{m}$ and a fill factor of $87 \%$. This device is a pure phase spatial light modulator (SLM) that allows the introduction of controlled linear phases. The reflected light goes back to the B-S and is redirected to the detector. As the lens (L) images the scene plane at the CCD plane, the linear phases addressed to the PA-LCoS generate subpixel displacements of the low-resolution images at the CCD plane. To this aim, the SLM phase-to-voltage response must be accurately calibrated by following some LCoS display calibration technique $[19,20]$. In addition, the relationship between the linear phase displayed at the PA-LCoS and the corresponding image displacement produced at the CCD plane must be calibrated as well. Once the sub-pixel displacements are controlled, a set of $n_{x} \cdot n_{y}$ sub-pixel 2-D displacements is generated with the SLM, where $n_{x}$ and $n_{y}$ are the numbers of sub-pixel displacements in $x$ and $y$ directions, respectively. In this situation, $n_{x} \cdot n_{y}$ images are recorded. The dimension of each raw image is $\left(s_{x}, s_{y}\right)$. Afterwards, raw images are properly interlaced in a larger image of dimension $\left(n_{x} \cdot S_{x}, n_{y} \cdot S_{y}\right)$. 


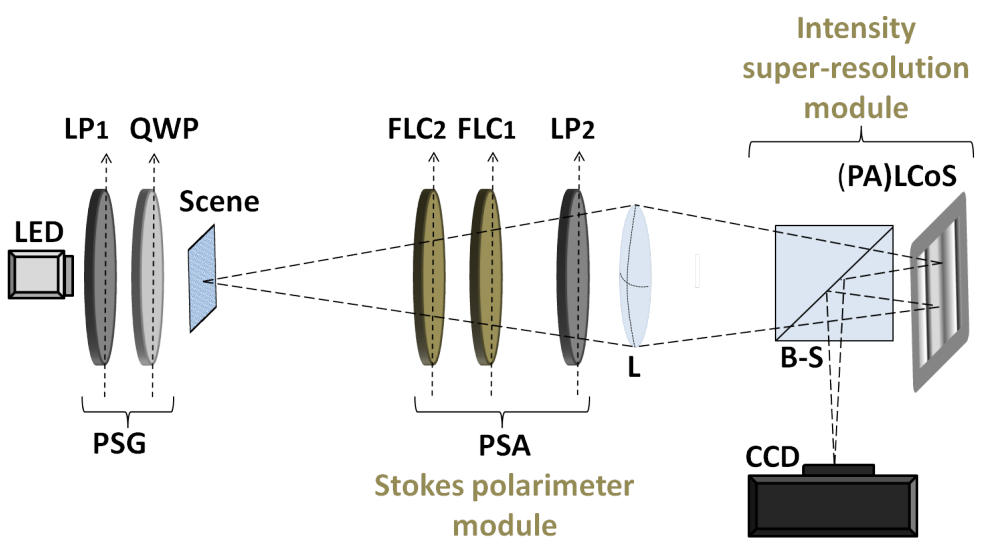

Fig. 8. Super-resolution imaging Stokes polarimeter set-up. This consists of two parts: an imaging Stokes polarimeter (two ferroelectric liquid crystal cells and a polarizer) and an intensity super-resolution module (LCoS spatial light modulator).

Note that all raw images suffer the intensity spatial average due to the pixel size, causing blurring effect. Thus, the final image of larger dimension, created by properly interlacing those raw images, will be consequently affected by the same blurring effect. From a mathematical point of view, the intensity detected by the camera can be described as the convolution of the scene intensity distribution $(I(x, y))$ with the shape of the camera pixel $(p(x, y))$ :

$$
I_{d}(x, y)=I(x, y) \otimes p(x, y)
$$

In order to remove this blurring effect, an inverse filtering process is applied in the frequency domain $[21,22]$. The goal of the algorithm is to isolate the intensity distribution $(I(x, y))$, eliminating as much as possible the effect of the pixel size $(p(x, y))$. To this aim, we first multiply the Fourier transform (FT) of the intensity detected by the camera $\left(I_{d}(u, v)\right)$ with the filter $(f(u, v))$, as shown in [22]:

$$
I_{\text {fittered }}(u, v)=I_{d}(u, v) \cdot f(u, v)=\frac{I_{d}(u, v) p^{*}(u, v)}{|p(u, v)|^{2}+\sigma},
$$

where $I_{\text {fittered }}(u, v)$ is the FT of the synthesized intensity image of the object, $p^{*}(u, v)$ and $|p(u, v)|$ are the complex conjugate and absolute value of the FT of the pixel shape respectively and, $\sigma$ is a parameter which takes small values and is used to avoid possible zeros at the denominator. In fact, $f(u, v)$ is the so-called Wiener filter [22], where we have considered that the images are mainly affected by white noise and the constant $\sigma$ is related with its spectral density.

Next, we perform the inverse Fourier transform of $I_{\text {filtered }}(u, v)$, obtaining the synthesized intensity image of the object. By applying this technique, we are able to obtain super-resolved images of the object because the blurring effect has been removed to certain extent.

Finally, by combining 4 super-resolved intensity images, and conducting the corresponding polarimetric data reduction calculation, a super-resolved polarization image is obtained.

In order to test experimentally the resolution enhancement in polarimetric images, a birefringence resolution target (Thorlabs model NBS 1963A) is used as a scene. It consists of a birefringent pattern sandwiched between two glass substrates. The pattern includes different groups of birefringent material, each one with different spatial frequencies but with a uniform transmission. Each group, sorted in two sets of five horizontal lines and five vertical lines, is labeled with a number referring to the cycles per millimeter.

The Stokes vector of the input object was measured when the scene was illuminated with linearly polarized light. Fig. 9 shows the Stokes vector elements obtained from regular polarization measurements (Fig.9(a,c,e,g)) and from the enhanced SR measurement (Fig. 9(b,d,f,h)). As expected, the object has uniform So, due to the uniform transmission of the pattern. Moreover, it is noticeable that the different frequency groups are only distinguishable through the S1, S2 and S3 channels, because of the non-uniform polarimetric spatial distribution. In general, it is observed that images obtained with the proposed technique offer much better resolution for the Stokes channels than the ones obtained from regular measurements. In order to show in more detail the resolution enhancement in the polarimetric images, the 
vertical cross sections corresponding to the black lines marked in Fig.9 are plotted in Fig.10. Note that cross sections of S1-S3 Stokes parameters in Fig.10 are zoomed in the $y$ axis including a range of 0.5 (the full Stokes scale goes from -1 up to 1). This is done to better visualize how the different frequency arrangements of interest are resolved by the experimental system.

(a)

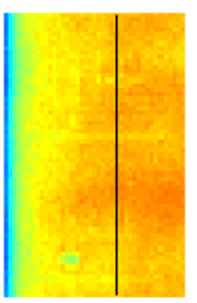

(e)

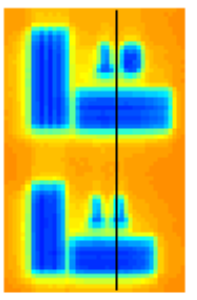

(b)

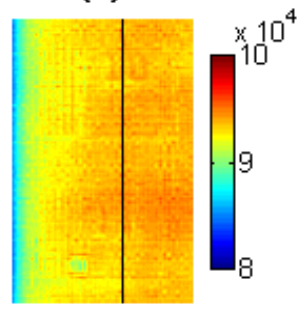

(f)

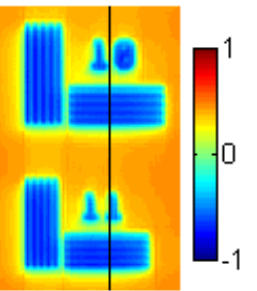

(c)

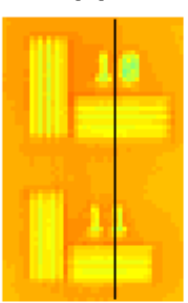

(g)

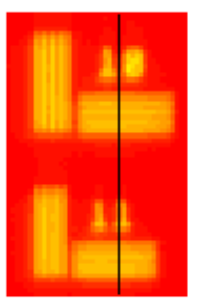

(d)

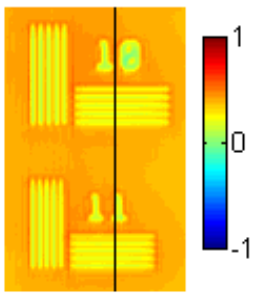

(h)

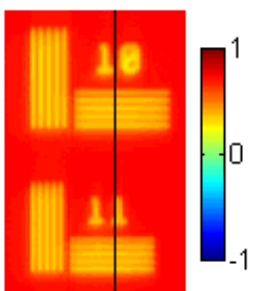

Fig. 9. Polarization image of a birefringent resolution test obtained by applying the two techniques. Regular resolution Stokes parameters: (a) S0, (c) S1, (e) S2 and (g) S3. Enhanced resolution Stokes parameters: (b) S0, (d) S1, (f) S2 and (h) S3.

It is observed that Stokes elements S1, S2 and S3 (Fig. 10(d,f,h), respectively) are much better resolved when applying the super resolution technique than the ones using the regular technique (Fig. 10(c,e,g), respectively). As an example, the dashed red circles in Fig.10 point out that whereas the 5 consecutive lines per group are clearly resolved when applying the enhancing SR algorithm, they cannot be resolved by applying a regular method.

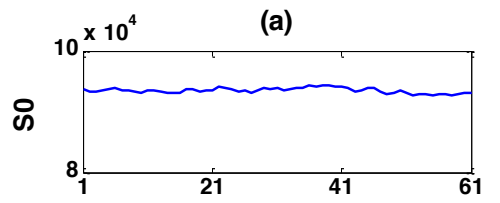

(c)

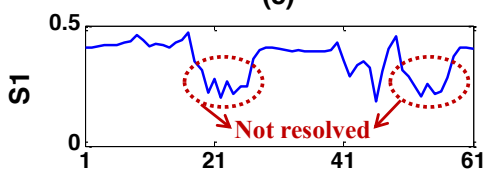

(e)

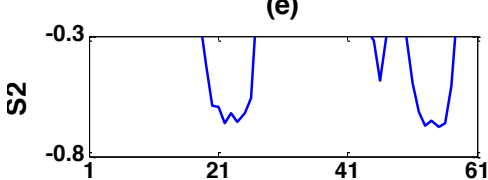

(g)

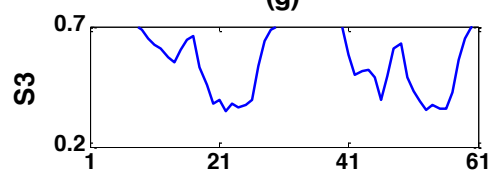

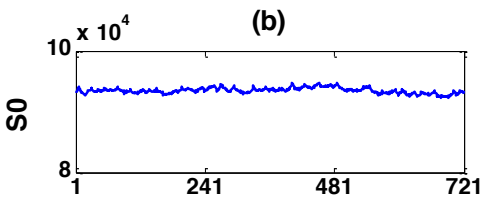

(d)

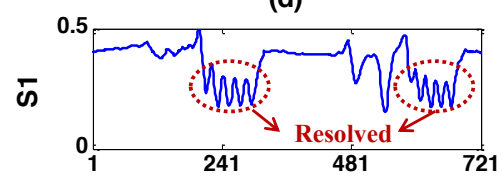

(f)

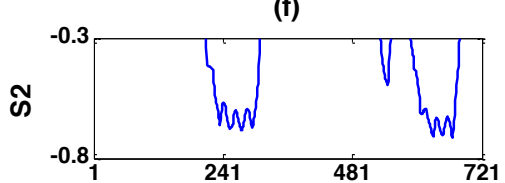

(h)

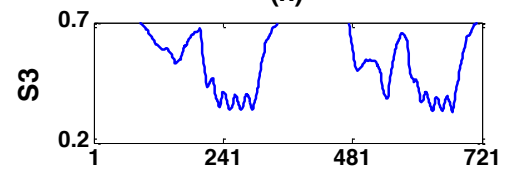

Fig.10. Cross section of the birefringent resolution test measurements shown in Fig.9. Regular resolution Stokes parameters: (a) $S_{0}$, (c) $S_{1}$, (e) $S_{2}$ and (g) $S_{3}$. Enhanced resolution Stokes parameters: (b) $S_{0}$, (d) $S_{1}$, (f) $S_{2}$ and (h) $S_{3}$. All Stokes elements are given as a function of the $y$ pixel position.

Figs. 9 and 10 represent a region of interest of the whole birefringent resolution target, chosen to highlight the resolution improvement that we have observed between both techniques. When the polarimetric images corresponding to the entire resolution test are analyzed, we observe that the largest resolved 
frequency in the best case ( $\mathrm{S} 1$ channel) reaches 9 cycles $/ \mathrm{mm}$ for regular imaging, whereas super-resolving imaging reaches 12.5 cycles $/ \mathrm{mm}$, leading to an improvement of resolution by a factor of 1.4. Certainly, the polarization measurement with enhanced resolution technique resolves intermediate states of polarization between two birefringent lines, whereas in regular images those regions are not resolved. Moreover, polarimetric data is given in multichannel images, thus, the contrast of these images depend on the channel analyzed, as it can be observed in Fig. 10. Particularly, S1 has larger contrast than S2 and S3 channels. This contrast not only depends on the original object, but also could depend on the coordinate system chosen to define the state of polarization. In addition, it will depend on the state of polarization of the beam illuminating the scene, which could be also optimized depending on the scene to be measured [23]. This study is an interesting perspective, although it is beyond the scope of this work.

\section{Conclusions}

First, a comparative analysis between four different polarimeters based on different materials exhibiting refractive index anisotropy was given: three types of liquid crystal cells (parallel aligned nematic, twisted nematic and ferroelectric) and biaxial crystals. The comparative table given in this work is a valuable summary for all those users who are interested in implementing a polarimeter based on liquid crystal cells or biaxial crystals.

Second, the implementation of an imaging polarimeter was detailed based on liquid crystal cells. Several polarization scenes were analyzed with the instrument, particularly non-uniform polarization distributions due to birefringence stress. Finally, we provided a new experimental configuration which combines an imaging Stokes polarimeter with an extra module for overcoming the spatial resolution limit due to the detector pixel size. Experimental results proved the improvement in the spatial resolution in polarization images by a factor of 1.4 .

\section{Acknowledgements}

This research was financially supported by the Spanish Ministerio de Ciencia e Innovación (BES-2010031696, FIS2009-13955-C02-01 and FIS2012-39158-C02-01). The author acknowledges to all coauthors that participated in all aforementioned studies, and especially to Dr. Juan Campos and Dr. Angel Lizana for their guidance during the elaboration of the doctoral thesis. 